\title{
Thoracoscopic visualization of a misdirected paravertebral catheter
}

\author{
Priscilla Phoon, MMed $\cdot$ Sze Ying Thong, MMed • \\ Puay Theng Koh, MRCS • Kenny Loh, MMed
}

Received: 22 February 2015/Accepted: 10 March 2015/Published online: 14 March 2015

(C) Canadian Anesthesiologists' Society 2015

A thoracic paravertebral catheter (Espocan ${ }^{\circledR}$, B. Braun
Meisungen AG, Germany) was inserted in a 42 -yr-old
female patient (who provided consent for publication of
this report) undergoing a thoracoscopic wedge resection of
the left upper lobe for a suspected lung metastasis. A $20 \mathrm{G}$
catheter was placed at the level of T6 through an 18G
Tuohy needle with the patient in the prone position. A parasagittal, ultrasound-guided, in-plane insertion technique was employed with a linear array probe (15$6 \mathrm{MHz}$, HFL 50) using a SonoSite Edge ${ }^{\circledR}$ ultrasound system (SonoSite, Bothell, WA, USA). The Tuohy needle was directed cephalad and the catheter was easily advanced

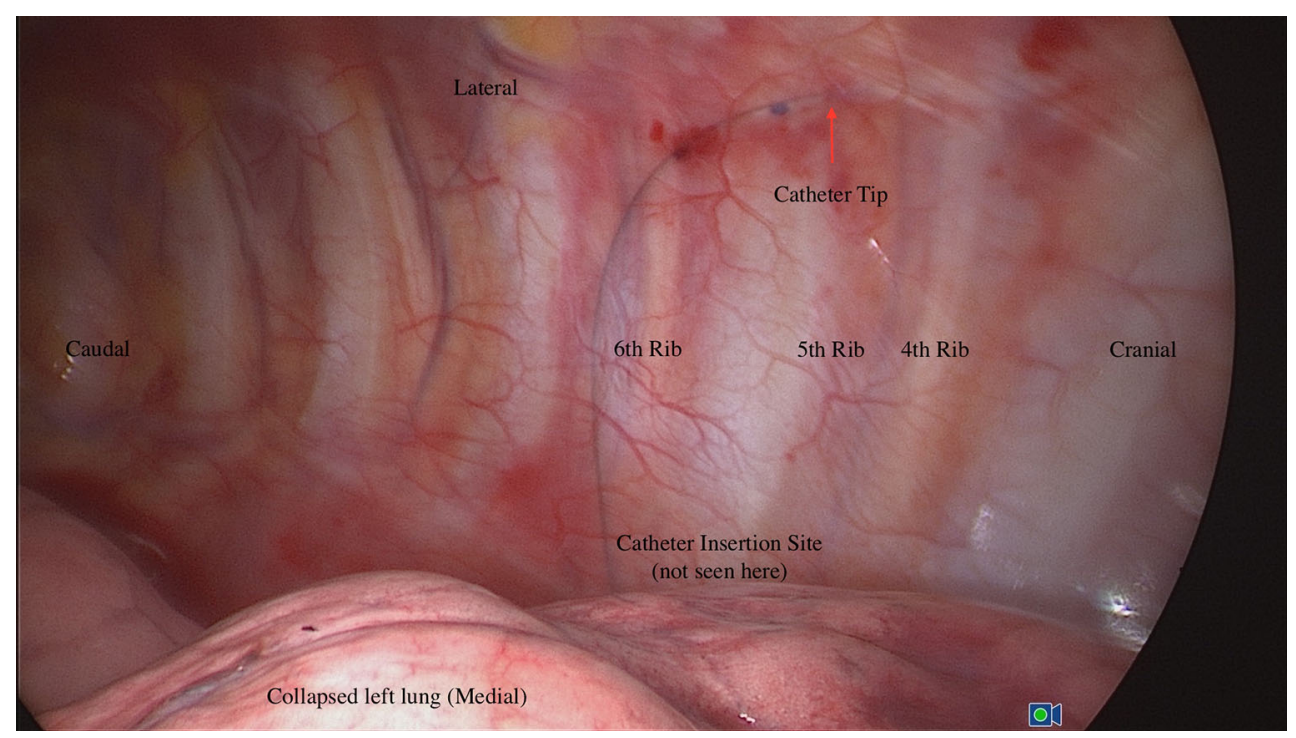

Figure Thoracoscopic view of a paravertebral catheter that has been misdirected through the paravertebral space and into the intercostal space with its tip (red arrow) located in the T5/T6 intercostal space

P. Phoon, MMed $(\bowtie) \cdot$ S. Y. Thong, MMed $\cdot$ K. Loh, MMed Department of Anesthesiology, Singapore General Hospital,

Singapore, Singapore

e-mail: priscilla.phoon.h.y@sgh.com.sg

P. T. Koh, MRCS

Department of Surgical Oncology, National Cancer Centre

Singapore, Singapore, Singapore and secured $5 \mathrm{~cm}$ past the needle tip. Next, $0.5 \%$ ropivacaine $10 \mathrm{~mL}$ was administered through the catheter and a T4-T8 sensory block to pinprick sensation was subsequently attained over the left hemithorax. During thoracoscopy, the catheter could be seen tracking in the T6T7 intercostal space then curling to lie in the T5-T6 space. Despite its lateral placement, a postoperative infusion of 
$0.1 \%$ ropivacaine at $7 \mathrm{~mL} \cdot \mathrm{hr}^{-1}$ maintained a left-sided T4T10 sensory block that provided 2 days of excellent analgesia.

The thoracic paravertebral space is bounded medially by the vertebral bodies, anterolaterally by the parietal pleural and innermost intercostal membrane, and posteriorly by the transverse processes, heads of the ribs and the superior costotransverse ligament. Our catheter has passed beyond the lateral border of the paravertebral space to lie between the intercostalis intimus and parietal pleura before curving underneath a rib in the extrathoracic space. We suggest that the thoracic paravertebral space may be a "potential" space rather than a discrete anatomical compartment. For those who consider this an intercostal catheter, the multilevel sensory block seen may be due to local anesthetic backtracking into the paravertebral space. This image illustrates the discrepancy between the insertion site and the final catheter position and may explain the variable spread of local anesthetic seen in some catheter-based techniques.

Conflict of interest None declared.

Funding No funding was required for the study. 